\title{
Behavioral Modulation Induced by Food Odor Aversive Conditioning and its Influence on the Olfactory Responses of an Oscillatory Brain Network in the Slug Limax marginatus
}

\author{
Tetsuya Kimura, ${ }^{1,4}$ Shoichi Toda, ${ }^{2}$ Tatsuhiko Sekiguchi, ${ }^{1}$ and Yutaka Kirino ${ }^{3}$ \\ ${ }^{1}$ Tsukuba Research Center \\ SANYO Electric Co., Ltd. \\ Tsukuba, Ibaraki 305, Japan \\ ${ }^{2}$ Faculty of Pharmaceutical Sciences \\ Kyushu University, \\ Hakuoka, Hakuoka 512, Japan \\ ${ }^{3}$ School of Pharmaceutical Sciences \\ The University of Tokyo, \\ Bunkyoku, Tokyo 113, Japan
}

\begin{abstract}
We compared behaviorally and physiologically the olfactory responses of slugs (Limax marginatus) that had been subjected to aversive, appetitive, or unpaired training with food odors (carrot or cucumber). In the aversive training, the slugs were exposed to the food odor as a conditioned stimulus (CS), and then quinidine sulfate solution as an unconditioned stimulus (UCS) was immediately applied to the lip of the slugs. This training caused a decrease in preference level for the CS. The unpaired training, in which the CS and the UCS were presented to the slugs with a 5-min interval, induced no change in the preference level for the CS. In the appetitive training, the slugs were allowed to eat the CS odor source without UCS application. When we used nonstarved slugs, it was found that the preference level for the CS increased upon the appetitive training. These results indicate that each training changed the preference for the odors in a characteristic manner. In the physiological experiments,
\end{abstract}

${ }^{4}$ Corresponding author. we used brain-inferior tentacular nose preparations isolated from slugs and investigated the olfactory responses of the oscillations in the local field potential (LFP) of the procerebral (PC) lobe. We found that odor presentation induced various types of changes in the LFP oscillation frequency, although the rate of occurrence of the frequency modulation differed between odors used in the aversive and the unpaired training (aversive-conditioned and unpaired odors). The aversive-conditioned odors induced a decrease in the oscillatory frequency. Unpaired odors did not change it. Moreover, odors used in the appetitive training (appetitive-conditioned odors) induced an increase in the frequency. Thus, it was considered that those modulations of PC lobe oscillatory activity were independent of odor and reflected learned preference for odors.

\section{Introduction}

Olfactory systems of many species of invertebrates and vertebrates have proven to be excellent models for the study of learning and memory. Odor recognition in mammalian olfactory bulbs is thought to involve both spatial and temporal patterns of oscillation amplitude modulation (Kauer 
Kimura et al.

1988) that are altered by odor learning (Freeman and Schneider 1982; Freeman and Skarda 1985). Some forms of synaptic plasticity in the olfactory bulb and piriform cortex have been demonstrated (Hasselmo and Bower 1990; Jung et al. 1990; Kanter and Harberly 1990; Sullivan et al. 1990). These experimental findings encourage the view that some aspects of the cellular substrate of odor identification and learning occur early in the olfactory pathways.

The central nervous system of the slug has also been considered to be a useful model system for investigation of associative learning, because slugs easily learn associations between applied odors and tastes and change their responses to conditioned stimuli (Sahley et al. 1981a; Sekiguchi et al. 1991). Various forms of classical conditioning (e.g., first order, second order, and presensory conditioning) have been reported to occur in slugs (Gelperin 1974; Sahley et al. 1981b; Suzuki et al. 1994). Furthermore, Chang and Gelperin (1980) showed that taste-taste associative learning occurred in isolated slug brain preparations that included the lip. However, the basic mechanisms of this classical conditioning in slugs are unclear. Many important questions remain, such as, Where are the odor memories stored? How are the odors represented in the CNS? and How does conditioning influence odor representation?

Recently, it was found that some interneurons within the procerebral (PC) lobe of Limax marginatus exhibit enhanced activity after odor aversive conditioning (Kimura et al. 1998a). For induction of this enhanced activity of the PC interneurons, associative (paired) presentation of a conditioned stimulus (CS) and an unconditioned stimulus (UCS) is needed (Kimura et al. 1998a). The finding suggests that the enhanced activity of the PC lobe plays a role in the acquisition of odor memories during odor aversive conditioning. Electrophysiological studies have been carried out to reveal the function of the PC lobe in odor recognition and categorization. Gelperin and Tank (1990) found that the PC interneurons of Limax maximus show spontaneous oscillatory activity. The PC oscillatory network is considered to be a coherent network constituted by numerous oscillators (Gelperin et al. 1993). It was also reported that odor stimuli applied to the superior tentacular nose modulated the oscillatory frequency and the coherency of the PC network (Gervais et al. 1996). These results also show the importance of the oscillatory network of the PC lobe in olfactory information processing.
In the present study, we observed the occurrence of behavioral modification by (food odor) aversive conditioning in Limax marginatus and then investigated its influence on the changes in the PC oscillatory activity to induce by olfactory stimuli.

\section{Materials and Methods}

\section{MATERIALS}

ANIMALS

Slugs (Limax marginatus) were maintained in our laboratory on frog chow (Oriental Yeast Co., Ltd.) with a light/dark cycle of $14: 10 \mathrm{hr}$ at $19^{\circ} \mathrm{C}$. Prior to the start of the experiments, each animal was isolated in a plastic chamber $(113 \times 105 \times 28$ $\mathrm{mm})$ for 2 days without food. The weight of the slugs used in the experiments was 1.5-2.0 grams before starvation. In the case of nonstarved slugs to be used for behavioral experiments, $500 \mathrm{mg}$ of frog chow was placed in the chamber daily.

\section{SOLLTIONS}

Carrot or cucumber juice was obtained by homogenization without dilution, followed by filtration through a filter paper (Yamada et al. 1992). The filtered extract was divided into $4-$ to $5-\mathrm{ml}$ aliquots in disposable tubes and stored in a freezer at $-30^{\circ} \mathrm{C}$. Each aliquot was used within $1 \mathrm{hr}$ after thawing. Frog chow solution ( $10 \%$ in distilled water) was also filtered and stored in the same manner. A saturated solution of quinidine sulfate in slug saline $\left(70.0 \mathrm{mu} \mathrm{NaCl}, 2.0 \mathrm{muKCl}, 4.9 \mathrm{mu} \mathrm{CaCl}_{2}\right.$, $4.6 \mathrm{mu} \mathrm{MgCl}_{2}$, 5.0mu glucose, 10 mм HEPES) was used as a UCS in aversive training

\section{CONDITIONING}

Each slug used in the behavioral and physiological experiments received one of three types of conditioning: differential, discriminative, or twoodor aversive conditioning.

DIFFERENTLAL CONDITIONING

Starved slugs received aversive training with either carrot or cucumber odor and unpaired training with the other food odor. In aversive training, each slug was placed on a clean glass plate 
$(30 \times 45 \mathrm{~cm})$ and kept without any stimuli for 5 min to acclimate to the environment. After acclimation, slugs usually began relatively straight crawling. The odor source used as a CS was a line of food juice $(10 \mathrm{~cm}$ in length) applied to the plate perpendicular to the body axis $0.5-1 \mathrm{~cm}$ in front of the head using a Pasteur pipette. About $10 \mathrm{sec}$ later, just before the slug touched the odor source, $100 \mu \mathrm{l}$ of quinidine sulfate solution as an aversive UCS was dropped onto the dorsoanterior surface of the animal. After a further $10 \mathrm{sec}$, the quinidine sulfate solution was washed off with slug saline, and the animal was returned to the holding chamber.

In unpaired training, a CS was also presented to a slug in the same way as in the aversive training. After $10 \mathrm{sec}$, the slug was returned to the holding chamber, and 5 min later it was placed on another clean glass plate and exposed to quinidine sulfate solution for $10 \mathrm{sec}$. Each slug received two trials in each training. The sequence of training was randomly chosen, and there was a $>20$-min intertrial interval.

\section{DISCRIMINATIVE CONDITIONING}

Starved slugs received aversive and appetitive training twice. The method for aversive training was as same as that described above. In appetitive training, carrot or cucumber odor as a CS was presented to the slugs as described above, but the animals were allowed to feed on the odor source (carrot or cucumber juice) for 5-10 min without application of quinidine sulfate. Each slug received two trials in each training. The sequence of training was randomly chosen with $>20$ min between trials.

TWO ODORS AVERSIVE CONDITIONING

Slugs received aversive training for both carrot and cucumber odors. Each slug received two conditioning trials for each odor. The sequence of training was randomly chosen with $>20 \mathrm{~min}$ between trials.

\section{BEHAVIORAL EXPERIMENTS}

One day after conditioning, a behavioral test was performed on each slug. Each animal was placed on a glass plate as described above and left there for $\sim 5 \mathrm{~min}$ to acclimate. After it began crawling straight, a line of odor source was placed 5-10 $\mathrm{cm}$ in front of it in the same manner as that for conditioning, and the behavior of each slug was observed for $5 \mathrm{~min}$. This experimental condition of the behavioral test was designed to make each slug choose between avoiding the odor source or approaching it. When slugs were attracted by the odor, they gradually approached the odor source and extended their lip toward it and then fed on it (eating behavior). On the other hand, when slugs avoided the odor source, they acutely changed the crawling direction within $1 \mathrm{~cm}$ from the applied odor source in many cases (avoidance behavior; see Fig. 2a, below). The influence of conditioning on the slugs' behavior was quantitatively analyzed in terms of a change the relative frequency of the occurrence of eating behavior obtained from 40 slugs, which had been subjected to a conditioning treatment. The preference of a group of slugs to an odor was evaluated by the frequency of the eating behavior with the odor, and the significance of the difference in the preference was analyzed by means of the $\chi^{2}$ test. The behavioral tests were performed by experimenters who did not know the treatments administered to each animal. In some cases, the slug mucus remaining on the experimental glass plate was traced onto a transparent film after the tests.

\section{PHYSIOLOGICAL EXPERIMENTS}

SCREENING OF ANIMALS FOR PHYSIOLOGICAL EXPERIMENTS

To minimize the influence of variation of conditioning performance among individual animals on the results of PC lobe response in physiological experiments, experimental animals were selected on the basis of the results of behavioral tests. For selection of experimental animals the following criterion was used: In differential- and discriminative-conditioned slugs, we used the slugs that avoided the odor source used in the aversive training three times or more in five tests with acute turning of the crawling directions (see Fig. 2a, below) and did not avoid the odor source used in the unpaired or the appetitive training. In two odors aversive-conditioned slugs, we used the slugs that avoided each CS in over three of five tests.

\section{PHYSIOLOGICAL EXPERIMENTS}

Each selected slug was anesthetized by injection of $200 \mu \mathrm{l}$ of $50 \mathrm{~mm} \mathrm{MgCl}_{2}$ in $10 \mathrm{~mm}$ HEPES buffer ( $\mathrm{pH} 7.4$ ), and the brain was exposed by cut- 
Kimura et al.

ting the dorsal head skin of the slug. Then the entire cerebral ganglion with right or left inferior nose (randomly selected) was gently dissected from the body. The dissected preparation was then placed into an experimental chamber filled with anesthetizing solution. The chamber consisted of two compartments with a slit connecting them (Fig. 1). After placement of the cerebral ganglion in the "ganglion compartment," the inferior nose was placed gently into the "nose compartment," with care being taken not to damage the nose and nerve tract (medial lip nerve) connecting the inferior nose and cerebral ganglion. The nerve was placed to pass through the slit. After placing the nose and ganglion, the anesthetizing solution was removed from both compartments, and the slit was sealed with Vaseline. Following this, both compartments were filled with normal slug saline. The local field potential (LFP) of the PC lobe was recorded through a suction pipette (tip diameter, 30 $\mu \mathrm{m})$ filled with saline. After putting the suction pipette on the posterior (cell mass) surface where the PC interneurons formed a layer of cell bodies, the solution in each compartment was perfused with normal saline (the flow rate, $1.5 \mathrm{ml} / \mathrm{min}$ ). The electrical activity from the PC electrode was filtered with a $0.08-\mathrm{Hz}$ low-cut and $100-\mathrm{Hz}$ high-cut passive filter and recorded in a PCM processing recorder. For analysis of the activity, the recorded data were transferred into a personal computer
(Macintosh 8100/80AV) via a 12-bit A-D converter with a 100-Hz sampling speed (MacAdiosII), and then the instantaneous frequency of each oscillatory cycle was calculated by detection of upper peaks of the oscillation with the use of a commercial wave analysis program (IgorPro, WaveMetrics, Inc.).

An odor was applied at a constant flow rate $(0.1 \mathrm{ml} / \mathrm{sec})$ through a glass pipette (Fig. 1, top) Five minutes before odor presentation, the olfactory epithelium of the inferior tentacle nose was exposed to flowing air to adapt to the constant airflow. Odor presentation was performed by changing the airflow route by alternate switching of two electric valves. Each valve was connected either to a control syringe containing a piece of paper moistened with water or a syringe containing a piece of paper moistened with $1 \mathrm{ml}$ of a CS odor source.

For the physiological study, we used brain-inferior tentacular preparations. This was based on our prior observation that induction of food avoidance by aversive-conditioned odors was more reliable in slugs possessing only the inferior tentacles than in the slugs possessing only superior ones (T. Kimura, unpubl.).

ANALYSIS OF ODOR RESPONSE

The period of every cycle was obtained by
Figure 1: A schematic illustration showing the setup used for electrical activity recording and odor stimulation. (Bottom) A typical trace of LFP recorded from a PC lobe. The LFP obtained from a PC lobe showed an oscillatory profile, and frog chow odor induced an increase in the oscillation frequency. The horizontal bar shows the stimulation period.
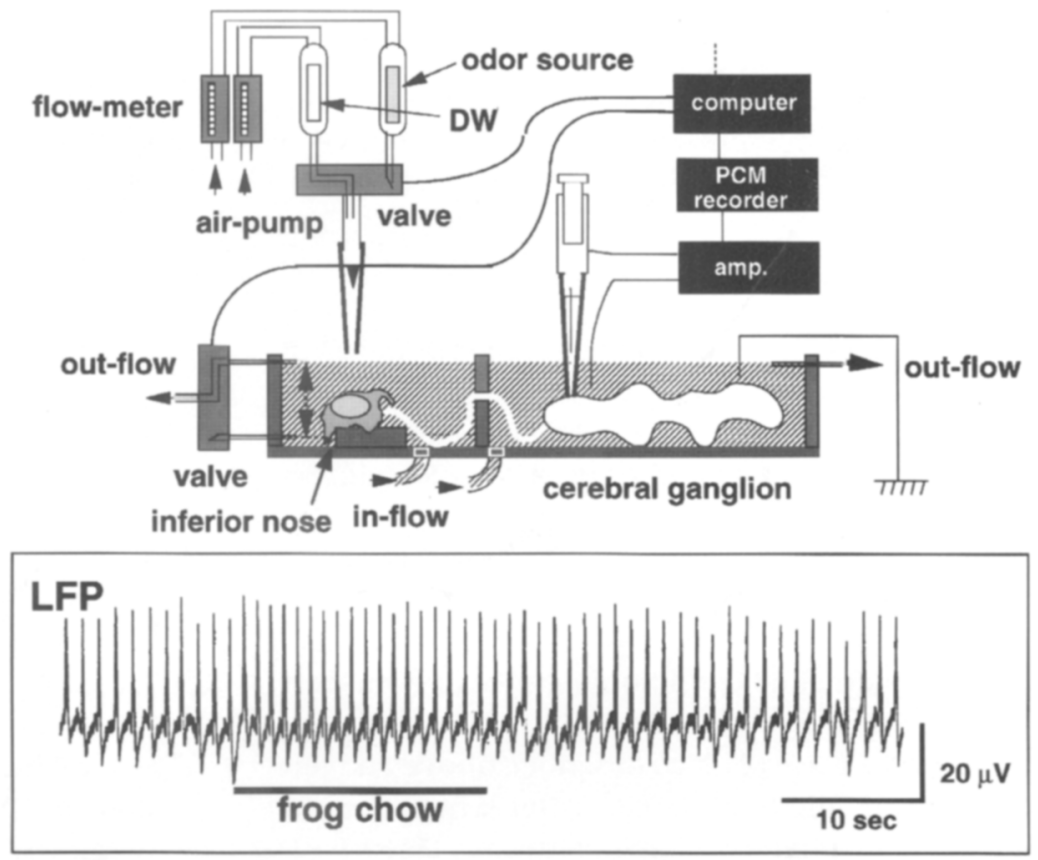
measuring a time from peak to peak, and the average cycle length (10 cycles) during stimulation was compared with that before stimulation (15-20 cycles) by means of the $t$-test. In the stimulation period, we ignored the first four cycles as a transient period and measured the fifth to the fourteenth cycle after the start of stimulation. Based on the analysis, the influence of the aversive and the unpaired training on the percentage of occurrence of statistically significant responses was analyzed by means of the $\chi^{2}$ test.

The major tendency of the frequency modulation induced by an odor (i.e., carrot, cucumber, aversive-conditioned, unpaired, or appetitive-conditioned odor) was determined based on a value showing relative change in oscillation frequency $(r \Delta F)$ obtained from each response of each slug, that was defined as follows: where $F_{\text {bef }}$ is the aver-

$$
r \Delta F=\frac{\left(F_{\text {stm }}-F_{\text {bef }}\right)}{F_{\text {bef }}}
$$

aged oscillatory frequency for 15-20 cycles of LFP oscillations just before stimulation and $F_{\text {stm }}$ is that for 10 cycles during odor presentation.

As an odor was presented more than twice, the averaged value of $r \Delta F$ for the responses was regarded as $r \Delta F$ of the preparation for the odor. The difference between the mean $r \Delta F$ s obtained from groups was evaluated by means of the $t$-test.

\section{Results}

\section{BEHAVIORAL CHANGES INDUCED BY ODOR CONDITIONING}

Prior to the physiological experiments, we performed behavioral tests to select the conditioned slugs. In the behavioral test, we observed the response of crawling animals to a line of odor source that was placed in front of the head. When naive slugs that had not been subjected to any treatments were attracted by the odor, they gradually approached the odor source and extended their lip toward it. In these cases, they always began active biting after touching the odor source. That type of behavior (eating behavior) was usually observed when carrot or cucumber juice as an odor source was presented to naive slugs (Fig. 2, naive). On the other hand, another type of re- sponse was often observed when an odor source used previously in aversive training was presented to slugs. In those cases, the slugs avoided the odor source by turning sharply when they reached a position located within $1 \mathrm{~cm}$ from the source (Fig. $2 \mathrm{a}, 2[\mathrm{CaQ}])$. Figure $2 \mathrm{~b}$ shows a typical example of results of the behavioral tests performed before the physiological experiments. Before conditioning, the slug showed a strong preference to both carrot and cucumber odors and showed the eating behavior in four of five tests when either of the odor sources was presented. After aversive training with carrot odor and unpaired training with cucumber odor (differential conditioning), the animal showed eating behavior in only one of five tests when carrot juice was presented although it showed the eating behavior in four of five tests when cucumber juice was presented (Fig. 2b).

The influence of the aversive conditioning on behavior in the groups of slugs (the number of slugs used in each group, $n=40$ ) was analyzed quantitatively. In the analysis, we counted the number of the slugs that showed the eating behavior in each observation period $(5 \mathrm{~min})$. In the differential-conditioned slugs, when slugs had been subjected to aversive training to the carrot odor and to the unpaired training with the cucumber odor ( $[\mathrm{CaQ}, \mathrm{Cu}-\mathrm{Q}])$, the preference for the carrot odor was significantly weaker than that for the cucumber odor (Fig. 3, $P<0.01, \chi^{2}$ test, left bars). Among the differential-conditioned slugs that had been subjected to the aversive training with cucumber odor and to the unpaired training with carrot odor ([Ca-Q, CuQ]), the preference to $\mathrm{cu}-$ cumber odor was lower than that to carrot odor (Fig. 3, $P<0.01, \chi^{2}$ test, right bars). Among two odors-conditioned slugs which had been aversively conditioned to both carrot and cucumber odors ([CaQ, CuQ]), the preference for each of the odors was weaker than that for the unpaired control odors in both of the differential-conditioned groups (Fig. 3, middle bars). These results demonstrate that our learning procedure effectively induced odor-taste associative learning in the slugs.

The influence of the appetitive conditioning treatment on the behavior was also examined using nonstarved slugs, of which the level of preference for the food odors was relatively lower than that of starved slugs. In this experiment, we compared the level of preference for the same odor (carrot) in three different groups, to eliminate the winfluence of possible differences in the level of preference for the carrot and cucumber odors (see 
a Naive (before conditioning)

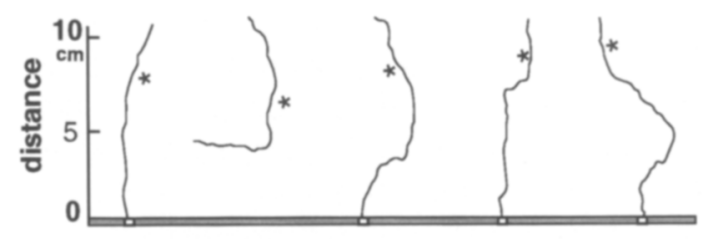

Conditioned [CaQ]

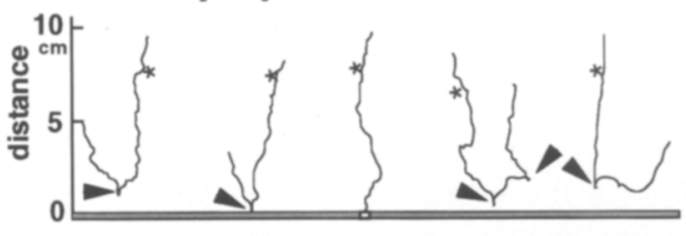

b

Naive (before conditioning)
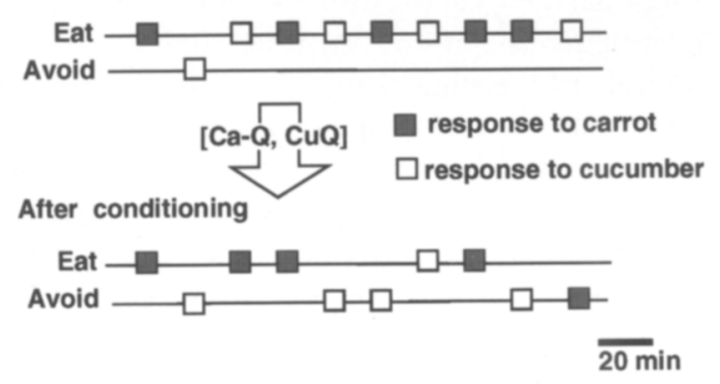

Figure 2: Behavioral changes induced by the aversive conditioning. (a) "Footprints" of a slug showing its behavior near the odor source before and after conditioning. In this experiment, the odor source (carrot juice) was placed on the plate $\sim 10 \mathrm{~cm}$ in front of a slug crawling forward. Exposure to the odor source usually did not induce stopping of the crawling before conditioning [each asterisk $\left(^{*}\right)$ shows the head position when the stimulus was presented] but induced extension of the lip and biting movements by the slug (eating behavior) in four of five tests. Each square shows the position at which biting movements were observed. After conditioning, the same slug turned sharply away from the odor source in four of five tests (arrowheads). (b) Ethograms showing response of a slug to odor sources: carrot (1) and cucumber ( $\square$ ) juice. Before conditioning, the slug usually approached both the carrot and the cucumber juice and exhibited eating behavior. After the behavioral test, the animal was subjected to the aversive conditioning to the carrot odor and to the unpaired procedure with the cucumber odor. After the treatment, the slug was repelled by carrot juice but not by cucumber juice. Such behavioral tests were performed before the physiological experiments for selection of experimental animals.
Fig. 4). The nonstarved slugs subjected to the appetitive training twice ([CaCa]; $n=30$ ) showed stronger preferences for the carrot odor than did the naive nonstarved slugs (naive; $n=30$ ) or the nonstarved slugs subjected to the unpaired training twice ( $[\mathrm{Ca}-\mathrm{Q}] ; n=30)\left(P<0.05, \chi^{2}\right.$ test). On the other hand, there was no significant difference in the level of preference for the carrot odor between the slugs that had been subjected to the unpaired training and the naive slugs $\left(P>0.05, \chi^{2}\right.$ test). These results show that the appetitive conditioning also induced a change in the preference level and that the unpaired control treatment did not. Such an influence of appetitive conditioning on behavior was not detected in the starved slugs (data not shown), apparently because they were strongly attracted by the food odors.

\section{FREQUENCY MODULATION INDUCED BY ODORS}

In the physiological experiments, we used 94 brain-nose preparations obtained from slugs that had been subjected to differential, discriminative, or two-odor aversive conditioning.

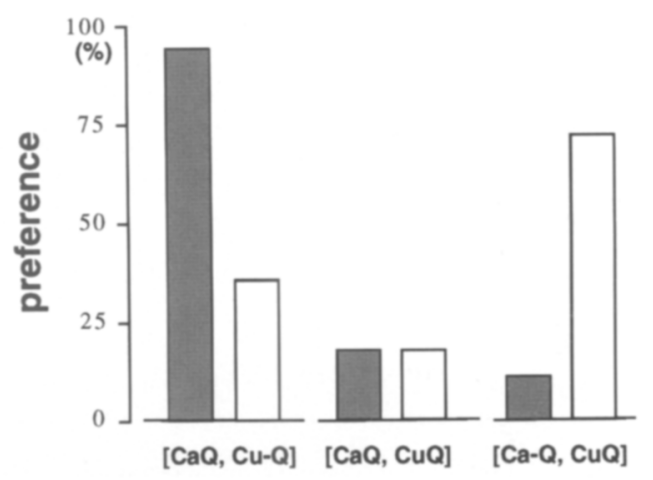

Figure 3: Effect of the aversive and unpaired training on slugs' preference to food odors. The preference to a CS was defined as the percentage of 40 slugs showing eating behavior when the CS odor source was applied. (2[CaQ, Cu-Q]) Responses of the slugs that had been subjected to the aversive conditioning to carrot odor and to the unpaired control treatment with cucumber odor. (2lCaQ, CuQ]) Response of the slugs that had been subjected to the aversive conditioning to both carrot (open bars) and the cucumber (solid bars) odor. (2[Ca-Q, CuQ]) Responses of the slugs that had been subjected to the unpaired control treatment with carrot and cucumber odor. The differential-conditioned slugs showed a significant difference in the frequency of occurrence of eating behavior for the presented odor source for the carrot and the cucumber odor $(2[\mathrm{CaQ}, \mathrm{Cu}-\mathrm{Q}]$ and $2[\mathrm{Ca}-$ $Q, C u Q 1 ; P<0.01$ in each, $\chi^{2}$ test). 


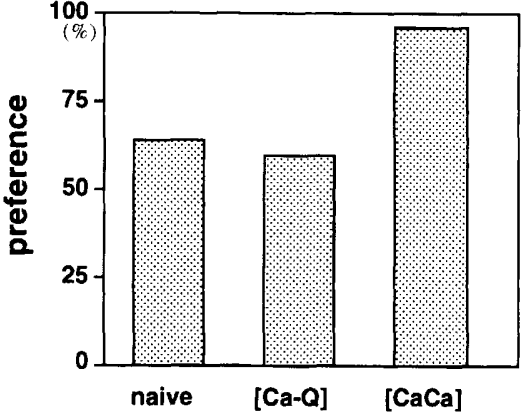

Figure 4: Influence of appetitive training on odor preference. In this experiment, we used nonstarved slugs, which showed a weaker preference for the carrot odor than did the starved slugs, to determine the influence of appetitive trainings on the level of preference (the frequency of occurrence of eating behavior elicited by the odor source). The preference for the carrot odor in the slugs subjected to the appetitive training (2[CaCa]; $n=30$ ) was significantly higher than that in the naive slugs $(n=30)$ (naive; $P<0.05, \chi^{2}$ test) or that in the slugs subjected to the unpaired control treatment $(n=30)\left(2[\mathrm{Ca}-\mathrm{Q}] ; P<0.05, \chi^{2}\right.$ test $)$.

The LFPs recorded from the PC lobe of Limax marginatus generally show an oscillatory pattern such as shown in Figure 1 (lower trace). The oscillation in LFP was strikingly similar to the PC lobe oscillation detected in Limax maximus by Gelperin and Tank (1990). Examples of the responses obtained from a preparation from a differentialconditioned slug are shown in Figure 5. When cucumber odor used in aversive training was presented to the inferior nose, we observed a slight but statistically significant decrease in the frequency of the PC oscillation $(P<0.05, t$-test $)$. The same preparation showed no change in the oscillation frequency upon the presentation of carrot odor used in unpaired training ( $P>0.05, t$-test). Furthermore, it showed a tendency toward an increase in the oscillation frequency by frog chow, although it was not statistically significant $(P>0.05, t$-test). We found no change in the amplitude of the PC oscillation in the responses. Although we did sometimes observe slight amplitude modulation accompanying the frequency modulation, in many cases the frequency modulation occurred without the amplitude modulation. On the basis of these observations, we focused on analysis of the frequency modulation following the odor presentation. In this analysis, the average cycle length during stimulation was compared with that before stimulation by means of the $t$-test.
In Table 1, we show the number of preparations that showed statistically significant changes

a
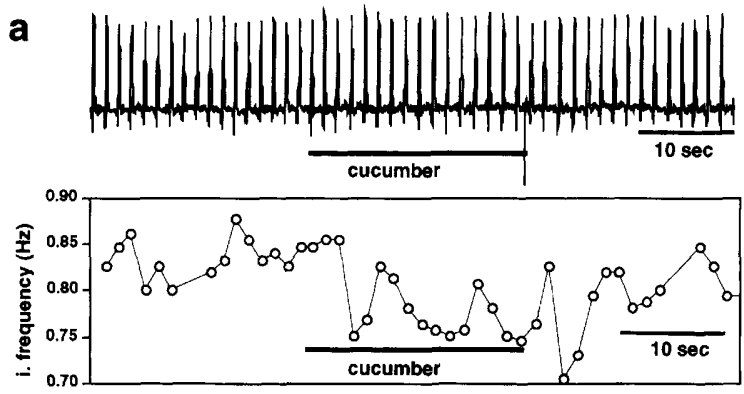

b
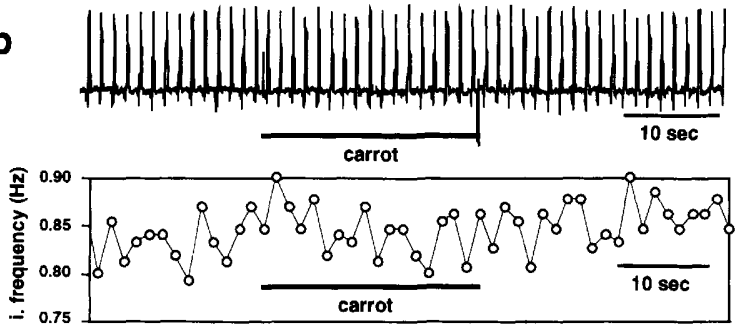

C
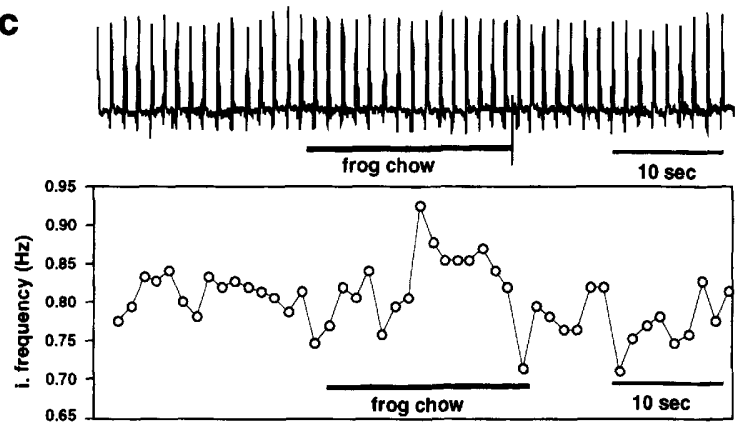

Figure 5: Odor-evoked modulation of PC lobe oscillation. The influences of cucumber, carrot, and frog chow odors on a PC lobe oscillation were examined using an differential-conditioned slug. (a) Response to aversiveconditioned carrot odor. The trace (top) shows the recorded LFP, and the graph (bottom) shows the instantaneous frequency change with time. Each horizontal bar indicates the stimulation period. Presentation of the aversive-conditioned carrot odor induced a change in the oscillation frequency from $0.834 \pm 0.024 \mathrm{~Hz}$ (mean \pm S.D., 20 cycles before stimulation) to $0.754 \pm 0.035 \mathrm{~Hz}$ (mean \pm S.D., in cycles $5-14$ after the onset of stimulation). The difference in the frequency was statistically significant $(P<0.01, t$-test). (b) Presentation of unpaired control cucumber odor resulted in no significant change in the oscillation frequency. (c) When frog chow, a daily food for the slug, was applied to the inferior tentacular nose, the frequency tended to increase, although there was no statistically significant difference $(P>0.05, t$-test). Based on these results, the preparation was defined as a sensitive one.

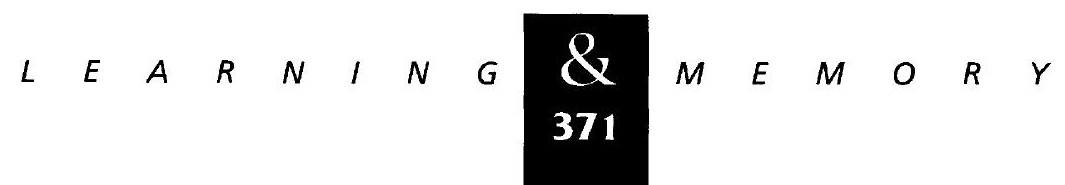


Kimura et al.

Table 1: Number of two trial aversive-conditioned, differential-conditioned, and discriminative-conditioned preparations that showed significant response to aversive-conditioned, unpaired, and appetitive-conditioned odor

\begin{tabular}{lccc}
\hline \multirow{2}{*}{ Group } & \multicolumn{2}{c}{ Number $(\%)$ of slugs responding to odors ${ }^{\mathrm{a}}$} \\
\cline { 2 - 4 } & \multicolumn{1}{c}{ aversive } & unpaired & appetitive \\
\hline Two trial aversive conditioned & $4(33) / 12^{*}$ & - & - \\
$\quad$ differential conditioned & $14(41) / 34^{*}$ & $3(9) / 34^{*}$ & - \\
discriminative conditioned & $16(33) / 48^{*}$ & - & $13(27) / 48^{*}$ \\
Total & $34(36) / 94^{*}$ & $3(9) / 34^{*}$ & $13(27) / 48^{*}$ \\
\hline
\end{tabular}

$\mathrm{a}\left({ }^{*}\right)$ Group size.

in oscillation frequency upon odor presentation. Significant responses to odors used in aversive training (aversive-conditioned odors) were found in 34 of the $94(36 \%)$ preparations. Responses to odors used in unpaired training (unpaired odors) were found in only 3 of the 34 (9\%) differentialconditioned preparations. There is a significant difference between the frequency of occurrence of a PC lobe response to the aversive-conditioned odor versus the unpaired odor; therefore, the frequency of occurrence of a significant response increased owing to aversive conditioning. In total, we found significant responses to either or both applied odors in 34 preparations and categorized them as sensitive preparations.

Odor presentation induced at least two types of changes in PC oscillation in the sensitive preparations (a decrease or an increase in frequency) (Figs. 1 and 5). We examined the differences in frequency modulation to carrot and cucumber odors in the sensitive preparations. In the sensitive preparations, aversive-conditioned cucumber odor induced a relative change in the oscillation frequency of $-6.52 \pm 4.37 \%$ (mean \pm S.D., $n=18$ ), and aversive-conditioned carrot odor induced a change of $-7.09 \pm 5.06 \%$ (mean \pm S.D., $n=16$ ). We found no statistically significant difference between the responses to the aversive-conditioned cucumber odor and the aversive-conditioned carrot odor (Fig. 6, $P>0.05, t$-test). The selection of sensitive preparations was not based on the polarity of the response (decrease or increase of frequency). Thus, it was considered that both aversive-conditioned odors showed the same tendency to induce a decrease in the frequency of PC oscillation. Based on this result, we calculated each relative change in the oscillation frequency without distinction between carrot and cucumber odor.
In the 15 sensitive differential-conditioned preparations, although the aversive-conditioned odors induce a change in the oscillation frequency of $-7.75 \pm 3.84 \%$ (mean \pm S.D.), the unpaired odors induced a change in the oscillation frequency of only $-1.05 \pm 1.92 \%$ (mean \pm S.D.) in the same preparations (Fig. 7). The difference in the frequency change was statistically significant caused by aversive and unpaired odors $(P \pm 0.05, t$-test $)$. This revealed directly that the unpaired odors did not induce a change in the oscillation frequency

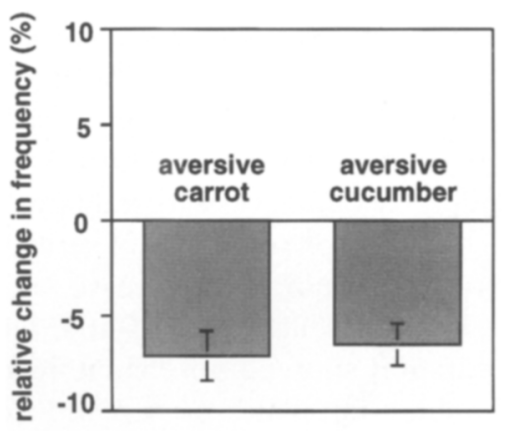

Figure 6: Comparison between the effect of aversiveconditioned carrot and cucumber odors on the oscillation frequency. Value of the frequency change in the PC oscillation was calculated from 20 cycles of LFP oscillations just before stimulation and 10 cycles (cycles 514 after the start of stimulation) during odor presentation (the mean frequency of the former was subtracted from that of the latter, and the result was divided by the mean frequency of the former) in each response obtained from the 34 sensitive preparations. Each column shows the mean value of the relative frequency change induced by the aversive-conditioned carrot or cucumber odor. We found no significant difference between the responses to the two odors in the relative change value of the oscillation frequency $(P>0.05, t$-test). Each error bar shows the S.E.M. 


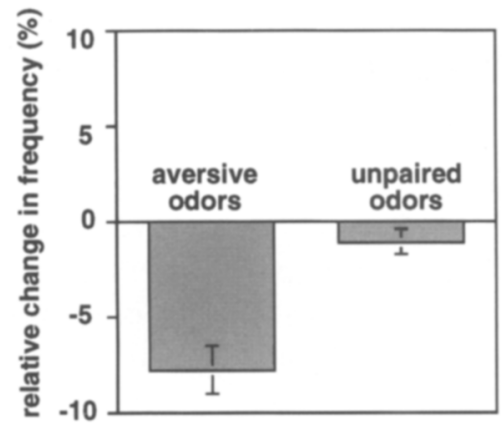

Figure 7: Comparison between the effect of the aversive-conditioned odors and the unpaired odors on the oscillation frequency in 12 sensitive differential-conditioned preparations. The aversive-conditioned odors induced a change in the oscillation frequency of $-7.75 \pm 3.84 \%$ (mean \pm S.D.) in mean, and the unpaired control odors induced one of $-1.05 \pm 1.92 \%$ (mean \pm S.D.) in mean. The difference between the responses to the odors was statistically significant $(P<0.01, t$-test). Each column shows the mean value of the relative frequency change induced by the aversive-conditioned odors or the unpaired control odors, and each error bar shows the S.E.M.

when the carrot or cucumber odor was used as the CS.

The influence of odors used in appetitive training (appetitive-conditioned odors) was also compared with that of the aversive-conditioned odors (Fig. 8). The change in the oscillation frequency induced by the appetitive-conditioned odors $(7.20 \pm 10.19 \% ; \quad$ mean \pm s.D. $)$ was significantly greater than not only that induced by the aversiveconditioned odors $(-6.53 \pm 3.47 \%$; mean \pm S.D. $)$ in the discriminative preparations $(P<0.01, t$-test $)$ but also that induced by the unpaired control odors in the differential-conditioned preparations $(P \pm 0.05, t$-test $)$. This shows that the three training procedures (aversive, unpaired, and appetitive training) caused different influences in the response of the PC oscillation.

\section{Discussion}

The present study revealed that the odors applied to the inferior nose modulated the frequency of PC oscillation and that the polarity of the odorevoked modulation altered according not to the kinds of odors but to whether prior training had been administered. These results strongly suggest that the PC lobe of $L$. marginatus plays an important role in cognition of previously experienced odors.
The PC lobe of slugs is a brain area speculated to play important roles in olfaction. The afferent fibers arising from the tentacle ganglia located at the superior and inferior tentacle tips directly terminate in the terminal mass of the PC lobe (Kimura et al. 1998a). Chase (1986) suggested that based on the results obtained using a 2-deoxyglucose technique, odor presentation enhances the activity of the PC lobe of Helix. On the other hand, Yamane et al. (1987) showed that protein phosphorylation was induced in Limax PC interneurons by 5-HT. It is reported that the NO cascade occurs in the PC lobe (Gelperin 1994; Gelperin et al. 1996). Both 5-HT and NO cascades are considered to be important in plastic changes at the level of synaptic efficiency (e.g., Hawkins et al. 1993; Robertson et al. 1994; Teyke 1996). Furthermore, it was reported that the metabolic activity of a localized cluster of Limax PC interneurons was enhanced when the slug showed odor aversive learning (Kimura et al. $1998 \mathrm{~b})$. These findings suggest the importance of the PC lobe not only in odor recognition but also in olfactory learning.

The aversive-conditioned odors mainly induced aversion to the odor source, and the appetitive-conditioned odors usually induced eating behavior (Fig. 3). Thus, the response of the PC lobe

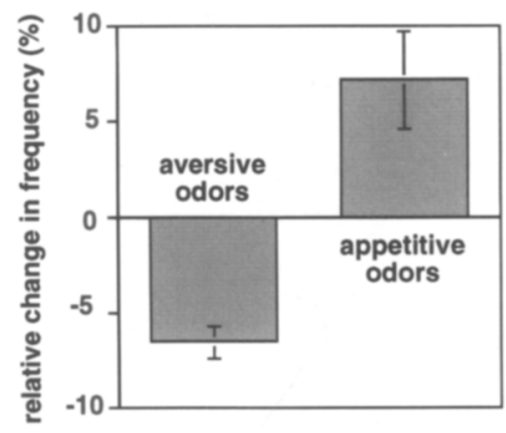

Figure 8: Comparison between the effect of aversiveconditioned odors and appetitive-conditioned odors in the oscillation frequency. The responses to the avesiveconditioned odors and to appetitive-conditioned odors in 18 sensitive discriminative preparations were analyzed for comparison of the stimulatory effects of the odors. The aversive-conditioned odors induced a change in the oscillation frequency of $-6.53 \pm 3.47 \%$ in mean, and the appetitive-conditioned odors induced one of $7.20 \pm 0.19 \%$ in mean. This difference was statistically significant $(P<0.01, t$-test). Each column shows the mean value of the relative frequency change induced by the aversive-conditioned odors or the appetitive-conditioned odors, and each error bar shows the S.E.M. 
to the odors was basically behavior oriented. The PC lobe may play an important role in determination of odor-elicited behavior. However, the unpaired control odors did not induce an increase in the frequency of PC oscillation (Fig. 7), whereas they usually induced eating behavior in the starved slugs used in the physiological experiments (Fig. 2). From only the results of the present study, we cannot explain the behavioral choice when the unpaired control odors or novel odors were presented to the inferior nose. It has already been suggested that novel odors presented to the superior nose induced a change in the frequency of PC lobe oscillation in several ways (Gervais et al. 1996), although the physiological meaning of the variations in the responses is unclear (Gervais et al. 1996). We consider that experiments performed using preparations that included both the superior and the inferior noses would be useful for explaining the behavioral effects of presentation of unpaired control or novel food odors.

When the carrot or cucumber odor was presented to the inferior nose, the frequency of the PC lobe oscillatory activity responds in three ways: no change, increase, or decrease. This may be accounted for by one of the following two possibilities: First, two types of modulatory fibers constitute the afferents, accounting for the frequency increase or decrease. If a certain plastic change in the level of synaptic efficiency (such as long-term depression or potentiation) had occurred in each of the terminals of the afferents because of conditioning, the aversive-conditioned odor would change the oscillation frequency of the PC lobe, with the magnitude of the change reflecting the proportion of the synaptic effects exerted via those afferents. However, we do not know that the olfactory afferents can be classified into two types based on the extent to which their activity affects the PC oscillation. The second possibility is that the odors induced a change in the level of neural activity related to the motivation for odor source aversion or eating, and this determined the response of the PC lobe. Gelperin et al. (1996) reported that some interneurons located in the buccal ganglion induced frequency modulation and obtained depressive inputs from the PC lobe. Yamada et al. (A. Yamada, T. Kimura, A. Iwama, Y. Suzuki, S. Kawahara, Y. Krino, and T. Sekiguchi, unpubl.) suggested that taste-evoked modulation of the PC oscillation was mediated by some metacerebral or mesocerebral neurons. It is likely that those types of neurons are related to the odor-in- duced frequency modulation. An optical recording study analyzing the frequency-decreasing response showed that the response to aversive-conditioned odors involved at least two different phases (Kimura et al. 1998b). In the early phase of the response, a depolarization occurred, and then hyperpolarization accompanied by a frequency decrease occurred. The early phase of the response is considered to be important for the odor recognition or categorization (Kimura et al. 1998b). If there would be feedback inputs to the PC lobe, the PC lobe activity in the late phase of response, in which the frequency modulation was observed, could be influenced by feedback activity and change from that in the early phase. Unfortunately, we cannot yet determine the mechanism producing the variety of responses. Currently, we are attempting to identify the feedback or output neurons with an intracellular recording technique to test the validity of the second possibility.

In recent years, oscillatory phenomena relating to recognition and/or learning processes have been detected in many species of vertebrates and invertebrates (e.g., Freeman and Schneider 1982; Eckhorn et al. 1988; Gray et al. 1989; Laurent and Davidowiz 1994; Laurent et al. 1996), and the studies in which they have been found have provided new viewpoints for encoding or information processing in the CNS. The PC lobe is an oscillatory network, the dynamics of which are influenced by prior experience. We hope that achieving an understanding of the PC oscillatory network in the slug brain will open new avenues for the study of learning and memory in animals.

\section{Acknowledgments}

We thank Alan Gelperin for comments on the manuscript. A part of this study was performed through Special Coordination Funds of the Science and Technology Agency and was supported by a grant from the Promotion of Basic Research Activities for Innovative Biosciences and by Grants-in-Aid for Scientific Research (0729105 and 07557143 to Y.K.) from the Minister of Education, Science, Sports, and Culture Japan.

The publication costs of this article were defrayed in part by payment of page charges. This article must therefore be hereby marked "advertisement" in accordance with 18 USC section 1734 solely to indicate this fact.

\section{References}

Chang, J.J. and A. Gelperin. 1980. Rapid taste-aversion learning by an isolated molluscan central nervous system. Proc. Natl. Acad. Sci. 77: 6204-6206. 
Chase, R. 1985. Response to odors mapped in snail tentacle and brain by $\left[{ }^{14} \mathrm{C}\right]$-2-deoxyglucose autoradiography. $]$. Neurosci. 5: 2930-2939.

Eckhorn, R., R. Bauer, W. Jordan, M. Brosch, W. Kruse, M. Munk, and H.J. Reitboeck. 1988. Coherent oscillations: A mechanism of feature linking in the visual cortex? Multiple electrode and correlation analysis in the cat. Biol. Cybern. 60: $121-130$

Freeman, W.J. and W.S. Schneider. 1982. Change in spatial patterns of rabbit olfactory EEG with conditioning. Psychophysiology 19: 44-56.

Freeman, W.J. and C.A. Skarda. 1985. Spatial EEG patterns, nonlinear dynamics and perception: The neo-sheintonian view. Brain Res. Rev. 10: 147-175.

Gelperin, A. 1974. Olfactory basis of homing behavior in the giant garden slug, Limax maximus. Proc. Natl. Acad. Sci. 71: 966-970.

1994. Nitric oxide meditates network oscillations of olfactory interneurons in a terrestrial mollusc. Nature 369: $61-63$.

Gelperin, A. and D.W. Tank. 1990. Odor-modulated collective network oscillation of olfactory interneurons in a terrestrial mollusc. Nature 345: 437-440.

Gelperin, A., L.D. Rhines, J. Flores, and D.W, Tank. 1993. Coherent network oscillations by olfactory interneurons: Modulation by endogenous amines. J. Neurophysiol. 6: 1930-1939.

Gelperin, A., D. Kleinfeld, W. Denk, and I.I.C. Cooke. 1996. Oscillations and gaseous oxides in invertebrate olfaction. J. Neurobiol. 30: 110-122.

Gervais, A., K. Kleinfeld, R. Delaney, and A. Gelperin. 1996. Central and reflex responses elicited by odor in a terrestrial mollusk. J. Neurophysiol. 76: 1327-1336.

Gray, C.M., P. Konig, A.K. Engek, and W. Singer. 1989. Oscillatory responses in cat visual cortex exhibit inter-columnar synchronization which reflects global stimulus properties. Nature 338: 334-337.

Hasselmo, M.E. and J.M. Bower. 1990. Afferent and association fiber differences in short-term potentiation in piriform (olfactory) cortex of the rat. J. Neurophysiol. 64: 179-190.

Hawkins, R.D., E.R. Kandel, and S.A. Siefelbaum. 1993. Learning to moderate transmitter release: Themes and variations in synaptic plasticity. Ann. Rev. Neurosci. 16: 625-665.

Jung, M.W., J. Larson, and G. Lynch. 1990. Long-term potentiation of monosynaptic EPSPs in rat piriform cortex in vitro. Synapse 6: 279-283.

Kanter, E.D. and L.B. Harberly. 1990. NMDA-dependent induction of long-term potentiation in afferent and association fiber systems of piriform cortex in vitro. Brain Res. 525: 175-179.

Kauer, J.S. 1988. Contribution of topography and parallel processing to odor conditioning in the vertebrate olfactory pathway. Nature 331: 166-168.

Kimura, T., H. Suzuki, E. Kono, and T. Sekiguchi. 1998a. Mapping of interneurons that contribute to food aversive conditioning in the slug brain. Learn. \& Mem. (this issue).

Kimura, T., S. Toda, T. Sekiguchi, S. Kawahara, and Y. Kirino. 1998b. Optical recording analysis of olfactory response of the PC lobe in the slug brain. Learn. \& Mem. (this issue).

Laurent, G. and H. Davidowiz. 1994. Encoding of olfactory information with oscillating neural assemblies. Science 265: 1872-1875.

Laurent, G., M. Wehr, and H. Dadowitz. 1996. Temporal representation of odors in an olfactory network. J. Neurosci. 16: 3837-3847.

Robertson, J.D., J. Bonaventra, and A.P. Kohm. 1994. Nitric oxide is required for tactile learning in Octopus vulgaris. Proc. R. Soc. Lond. B 256: 268-273.

Sahley, C., A. Gelperin, and J.W. Rudy. 1981a. One-trial associative learning modifies food odor preferences of a terrestrial mollusc. Proc. Natl. Acad. Sci. 78: 640-642.

Sahley, C., J.W. Rudy, and A. Gelperin. 1981b. Analysis of associative learning in a terrestrial mollusc. J. Comp. Physiol. 144: $1-8$.

Sekiguchi, T., A. Yamada, H. Suzuki, and A. Mizukami. 1991. Temporal analysis of the retention of a food aversive conditioning in Limax fluvas. Zool. Sci. 8: 103-111.

Sullivan, R.M., D.A. Wilson, R. Wong, A. Correa, and M. Leon. 1990. Modified olfactory bulb responding to learned natural and artificial material odors. Dev. Brain Res. 53: $243-247$.

Suzuki, H., T. Sekiguchi, A. Yamada, and A. Mizukami. 1994. Sensory preconditioning in the terrestrial mollusk, Limax flavus. Zool. Sci. 11: 121-125.

Teyke, T. 1996. Nitric oxide, but not serotonin, is involved in acquisition of food-attraction conditioning in the snail Helix pomatia. Neurosci. Lett. 206: 29-32.

Yamada, A., H. Suzuki, T. Sekiguchi, and A. Mizukami. 1992. Behavioral analysis of internal memory state using cooling induced retrograde amnesia in Limax flavus. ). Neurosci. 12: 729-735.

Yamane, T., A.B. Oestericher, and A. Gelperin. 1987. Serotonin-stimulated biochemical events in the procerebrum of Limax. Cell. Mol. Neurobiol. 9: 447-459.

Received May 23, 1997; accepted in revised form January 12, 1998.

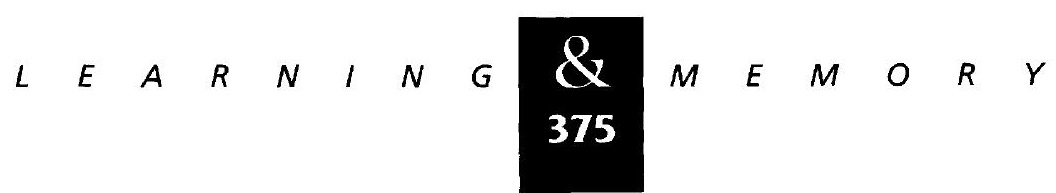




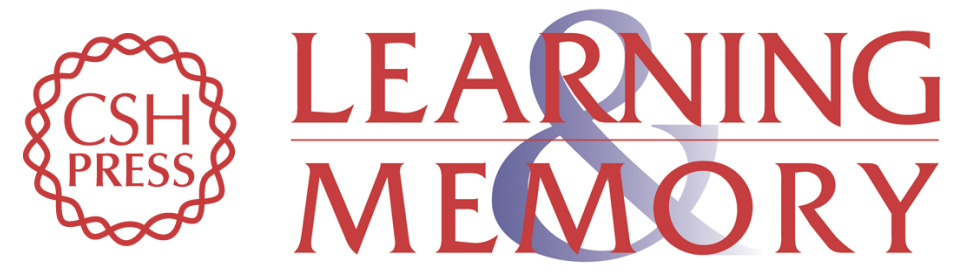

\section{Behavioral modulation induced by food odor aversive conditioning and its influence on the olfactory responses of an oscillatory brain network in the slug Limax marginatus.}

T Kimura, S Toda, T Sekiguchi, et al.

Learn. Mem. 1998, 4:

Access the most recent version at doi:10.1101//m.4.5.365

References This article cites 28 articles, 7 of which can be accessed free at: http://learnmem.cshlp.org/content/4/5/365.full.html\#ref-list-1

License

Email Alerting

Receive free email alerts when new articles cite this article - sign up in the box at the Service top right corner of the article or click here. 